\title{
MASS LOSS RATES IN CENTRAL STARS OF PLANETARY NEBULAE
}

\author{
M. Perinotto \\ Istituto di Astronomia, Università di Firenze, Italy
}

\section{ABSTRACT}

Central stars of planetary nebulae (PNCS) frequently exhibit fast winds (cf. Cerruti-Sola and Perinotto, 1985; C.P. and Grewing, this volume). They may be important for the structure of the whole nebula as well as for the evolution of the central star. Their speed is typically two orders of magnitude higher than that of the classical optically visible nebula, which in turn expands a few times faster than the most external winds detected in few cases in the radio domain.

I review here the status of art in the determination of the mass loss rates $(\dot{M})$ associated with these fast winds. I restrict myself to the 'observational' determinations. Only at the end I will say something about the predictions of multi-scattering line radiation wind driven theory in connection with one best studied object: N6C 6543. This allows one to conclude that this theory may be the right explanation also for these winds.

\section{INTRODUCTION}

The mass loss rates associated with a stellar wind might be obtained by using: i) P Cygni profiles of lines of heavy ions, ii)P Cygni-like profiles of hydrogen or helium lines, iii) free-free radiation in the infrared or iv) free-free radiation in the radio domain. Use of iii) and iv) has not yet successfully performed in PNCS because of the faintness of the radiation to be measured and of difficulties of taking into account the nebular contamination. Use of ii) has been done so far only in one or two objects. Thus the available $\dot{M}$ in PNCS almost all come from i).

Since various strong lines of heavy ions fall in the UV range observed by the IUE satellite ( $\lambda 1200-3200 \mathrm{~A})$, the observational basis for the existing values of $\dot{M}$ in PNCS rests almost exclusively in IUE data. These consist of low $(6 \mathrm{~A})$ and high $(0.15 \mathrm{~A})$ spectral resolution spectra, taken with a large aperture (oval $\approx 10 " x 20 "$ ) or a small aperture (circular, 3" in diameter). The low resolution spectra are adequate to reveal P Cygni-type profiles, but fail if the profile is essentially in emission, as in Wolf-Rayet stars, or is dominated by a blue-shifted absorption (cf. CP). They are also useful for measuring the edge velocity associated with the wind and for first approximation determination of $\mathrm{M}$. The high resolution spectra are very appropriate to study in detail the whole profile, but they can be used only in objects bright enough to give a measurable stellar continuum.

The lines in which P Cygni profiles have been seen in PNCS are the resonance lines NV $\lambda$ 1238.82, 1242.82; CIV 1548.20, 1550.77; SiIV 1393.73, 1402.73, the subordinate lines OIV 1338.60, 1342.98, 1343.51; OV 1371.29; NIV 1718.55 and the recombination line $\mathrm{He}$ II $1640.5 \mathrm{~A}$.

\section{METHODS TO INTERPRET THE P CYGNI PROFILES OF HEAVY IONS LINES}

The methods developed to interpret these lines are: i) the escape probability method (EP), ii) the first moment of flux distribution method (FM), iii) the comoving frame method (CF), and iv) the SEI method (Sobolev plus exact integration). 
In method i), the transfer equation of pure scattering line is solved using the escape probability formalism in the frame of the observer (Castor, 1970), under the Sobolev approximation. The last requires that in each point of the wind the macroscopic velocity is much larger than the stochastic velocity (local turbolence plus thermal motions). That is largely true across most of the winds in PNCS. Based on this method, Castor and Lamers (1979) have produced an atlas of theoretical profiles which has been used even in PNCS. The method has been reconsidered for the case of the excited lines and for an easier handling of the doublets by Olson $(1981,1982)$ respectively.

Method ii) has been developed by Castor, Lutz and Seaton (1981; CLS) just for use with the low resolution IUE data. It requires measuring the first moment $\mathrm{W}_{1}$ of the flux distribution across the profile. The assumptions are: a) Sobolev theory of pure scattering line formation for a two-level atom in an expanding atmosphere and b) simple expressions (found successful in early type $O B$ stars) for the velocity and opacity laws in the wind. Surdej (1982) has further investigated the CLS method showing that $\mathrm{W}_{1}$ is actually independent of the velocity and opacity laws and of other costraints. However this is true only for very optically thin lines. Otherwise with the CLS method one underestimates $\dot{M}$. Surdej (1983) and Hutsemékers and Surdej (1987; HS) have proposed a modification of the CLS method, for resonance and subordinate lines respectively. These improvements are useful when $\mathrm{W}_{1}>0.24$ or $\mathrm{W}_{1}>0.01$ respectively. We call this modified FM method FMm. The FM method has been used extensively in PNCS.

In iii) the radiative transfer equation of the line is solved in the frame of the moving fluid, under the Sobolev approximation (Lucy, 1971) or free from this assumption (Mihalas, et al. 1975). The last approach has been worked out by Hamann $(1980,1981)$. Based on this work, Schonberg (1985 a) has published an atlas of theoretical profiles of single resonance lines. Only one PNCS (NGC 3242) has been studied so far with the Hamann's procedure. Recently various authors have started to drop the 2-level atom approximation, used in the so far mentioned methods, for the line formation in the expanding atmosphere, introducing multi-level atoms. That may result particularly relevant for the subordinate lines observed with IUE. These lines have been treated in multi-level atom by Wessolowski and Hamann (1986). Applications to specific PNCS have not yet appeared.

Method iv), the SEI method, has been worked out by Lamers et al (1987). The source function is calculated in the Sobolev approximation, while the formal solution of the transfer equation is calculated exactly. Collisional effects are taken into account as well as the presence of a turbulent velocity across the wind. In addition the possible existence of a photospheric absorption at the base of the wind is allowed for. This method, as just illustrated, or in a less complete version (Bombek et al, 1986; BKB) has been used so far in a few PNCS. I call the method of BKB the SEIs method (SEI simplified).

\section{MERITS OF THE METHODS}

Method i). The EP method was found to reproduce satisfactorily the observed P Cygni profiles of thin lines in population I OB stars. At increasing optical depths, large deviations exist across the whole profile. In particular in the intermediate and inner parts of the wind, the theoretical profiles are unable to reach the observed depth of the absorption. Indeed by comparing the theoretical profiles of the EP method with those obtained with the more powerful CF method, it has been shown (Hamann, 1981; 
Schonberg,1985 b) that the EP method works well for optically thin lines formed under very large velocity gradients in the wind. The profiles of the EP method differ instead substantially from those of the CF method in other cases.

Method ii). The main limitation lies in the assumed velocity and opacity laws, which may not be the same in all objects.

Method iii). The CF method is the best so far developed, because it avoids the Sobolev approximation. As a consequence, it is accurate also for low velocity winds. However Hamann has used a pure scattering line source function, therefore with occupation numbers governed only by radiation. This is true of all the studies of winds in PNCS made with the methods so far mentioned. This limitation may be not of a minor importance in the applications to PNCS, as we will see later on.

Method iv). The principle of the SEI method has been suggested by Hamann (1981). Lamers et al. (1987) have worked it out in a practical way, including various physical effects, in particular the collisions. Schonberg (1985b) has discussed the validity of the basic assumptions of the SEI method finding that it becomes inaccurate at low wind velocities or very high optical depths. On the other hand, Lamers et al. have proved that the SEI's profiles agree very chsely with the corresponding ones of the CF method in all cases published by Hamann (1981), except for a small zone close to the center of the profile. These are cases of high ratio of the wind to the stochastic velocity, appropriate to the situation in PNCS.

\section{PRACTICAL CALCULATION OF $\dot{M}$}

With methods EP, CF and SEI one compares the observed profiles with theoretical ones computed for assumed parametrizations of the velocity and opacity laws. Usually one parameter, $\beta$, describes the velocity law and two parameters, $\gamma$ and $T$ (the total optical depth) describe the opacity law. In the SEI method other parameters are present. In particular the role of the collisions is specified by the parameter $\varepsilon$. When the best fit is obtained for a given set of the parameters, the mass loss rate follows from:

$$
\mathrm{q}_{\mathrm{i}} \dot{\mathrm{M}} \propto \mathrm{R}_{*} \mathrm{v}_{\infty}{ }^{2} \mathrm{f}(\mathrm{w}) \beta, \gamma, \mathrm{T}, \varepsilon \quad \exp \left(\mathrm{E}_{\mathrm{gl}} / \mathrm{kT}_{\mathrm{rad}}\right) / \mathrm{A}_{\mathrm{el}}
$$

where $R_{*}$ is the stellar radius, $q_{i}(w)$ is the fractional abundance of the relevant ion, $A_{e l}$ the elemental abundance, $f(w)$ a function of the normalized velocity $w=v(r) / v_{\infty}$, which is known when the parameters are specified, $\mathrm{E}_{\mathrm{gl}}$ the energy of the transition between the ground level and the lower level of the observed transition and $T_{\text {rad }}$ the radiation temperature at the frequency corresponding to $\mathrm{E}_{\mathrm{gl}}$. Clearly $\mathrm{E}_{\mathrm{gl}}=0$ for resonance lines. In the case of the FM method, the quantities $\mathrm{q}_{\mathrm{i}}(\mathrm{w})$ and $\mathrm{f}(\mathrm{w})$ in equation (1) are to be substituted with $\left\langle\mathrm{q}_{\mathrm{i}}\right\rangle$ and $\mathrm{W}_{1}$, the average ionization fraction of the element and the observed first moment of the flux distribution, respectively. This in the CLS formulation. In the HS formulation one has to use instead of $\mathrm{W}_{1}$ a function of $\mathrm{W}_{1}$, which, for $\mathrm{W}_{1}$ greater than the above mentioned values, depends on the velocity and opacity laws at work in the wind.

\section{DETERMINATION OF MASS LOSS RATES IN PNCS}

I summarize in Table 1 the available determinations of $\dot{\mathrm{M}}$ in PNCS. The values 
range from $510^{-11}$ to $210^{-6} \mathrm{M}_{\mathrm{O}} \mathrm{yr}^{-1}$, with a large scatter among the determinations of different authors in the same objects. One should bear in mind that among different authors there are not only differences in the methods but even in the way of computing $q_{i}$ and in the adopted fundamental stellar quantities appearing in equation (1). I will come later on to this point. From the study of HS (see before) one infers that the determinations by CP are likely to be underestimated by factors $2-3$. The values obtained from high resolution data are often larger than those obtained with low resolution data by factors much larger than the above. We cannot however assert that the first ones are automatically the more correct. All the individual determinations of $\dot{M}$ in PNCS are presented in a $\log \dot{M}\left(M_{0} \mathrm{yr}^{-1}\right), \log \left(\mathrm{L} / \mathrm{L}_{\mathrm{o}}\right)$ diagram in Fig. 1, together with the same numbers for population POB stars (Garmany and Conti, 1984). The determinations belonging to the same central star have been joined by straight lines. The scatter among individual determinations in the same object is very large not only in $\dot{M}$ but even in the luminosity.

TABLE 1- Mass loss rates in PNCS

\begin{tabular}{llrl} 
Object & \multicolumn{1}{l}{$\log (\dot{\mathrm{M}})$} & \multicolumn{1}{l}{ Object } & \multicolumn{1}{l}{$\log .(\dot{\mathrm{M}})$} \\
& & & \\
NGC 40 & $-7.52:(1)$ & NGC 6891 & $-9.22(1)$ \\
1535 & $-9.00(1) ;-7.00(2) ;-7.2(3)$ & 7009 & $-9.52(1) ;-8.0(3)$ \\
2371 & $-7.30:(1) ;<-7.00(4)$ & IC 418 & $-8.40:(1) ;-7.2(3) ;-6.52(11)$ \\
3242 & $-10.10 \div-8.10(5) ;-10.3(3)$ & 2149 & $-7.00(1) ;-8.00(12) ;-6.7(3)$ \\
5189 & $-8.22(1)$ & 3568 & $-8.30(1) ;-8.40(13)$ \\
6210 & $-9.22(1) ;-6.8(3) ;-7.92(6)$ & 4593 & $-8.00(1)$ \\
$6543-7.05(7) ;-6.15(8) ;-6.49(9) ;$ & A 30 $-9.52(1)$ \\
$-5.77(6) ;-7.49(10)$ & A 78 $-9.05:(1)$ \\
6572 & $-9.00(1)$ & Hu 2-1 $-8.05:(1)$ \\
6826 & $-7.70(1) ;-5.8(3) ;-6.82(6) ;$ & Sw St $1-7.30(1) ;-7.19(14)$ \\
$-7.66(10)$ & K 648 & $-9.70(15)$
\end{tabular}

Sources (authors, method): 1 (CP, FM); 2 (AK, EP); 3 (BKB, SEIs); 4 (PGGW, EP); 5 (HKMP, CF); 6 (HS, FMm); 7 (CLS, FM); 8 (He, EP); 9 (BCG, EP); 10 (PCL, SEI); 11 (CBBG, No details); 12 (PBC, EP); 13 (H, EP); 14 (FV, optical); 15 (ASHAW, EP). Low resolution: $1,6,7,15$. High resolution: the others except 14 (optical recombination line).

\section{COMPARISON WITH THEORETICAL PREDICTIONS OF $\dot{\mathrm{M}}$}

In Fig. 1 the theoretical predictions of the single-scattering line radiation wind driven theory for population I OB stars by Friend and Abbott (1986) and for PNCS by Pauldrach et al. (1987) are also shown. We see that the predictions of the theory are not far from the area where the determinations fall. However, before a close comparison with the predictions of the radiation driven theory can be made in PNCS, one must first discuss the reliability of the various experimental determinations. 


\section{RELIABILITY OF EXPERIMENTAL $\dot{M}$ IN PNCS}

In addition to errors due to inaccuracy of the methods used to interpret the lines, errors in $\dot{M}$ come from the evaluation of $q_{i}$ and of the stellar parameters entering into eq. (1). To have $q_{i}$ one needs a theory of the ionization equilibrium in stellar winds complete with all the relevant physical processes: radiation, collisions and Auger ionization in multi-level atoms. Such a theory is not yet available.

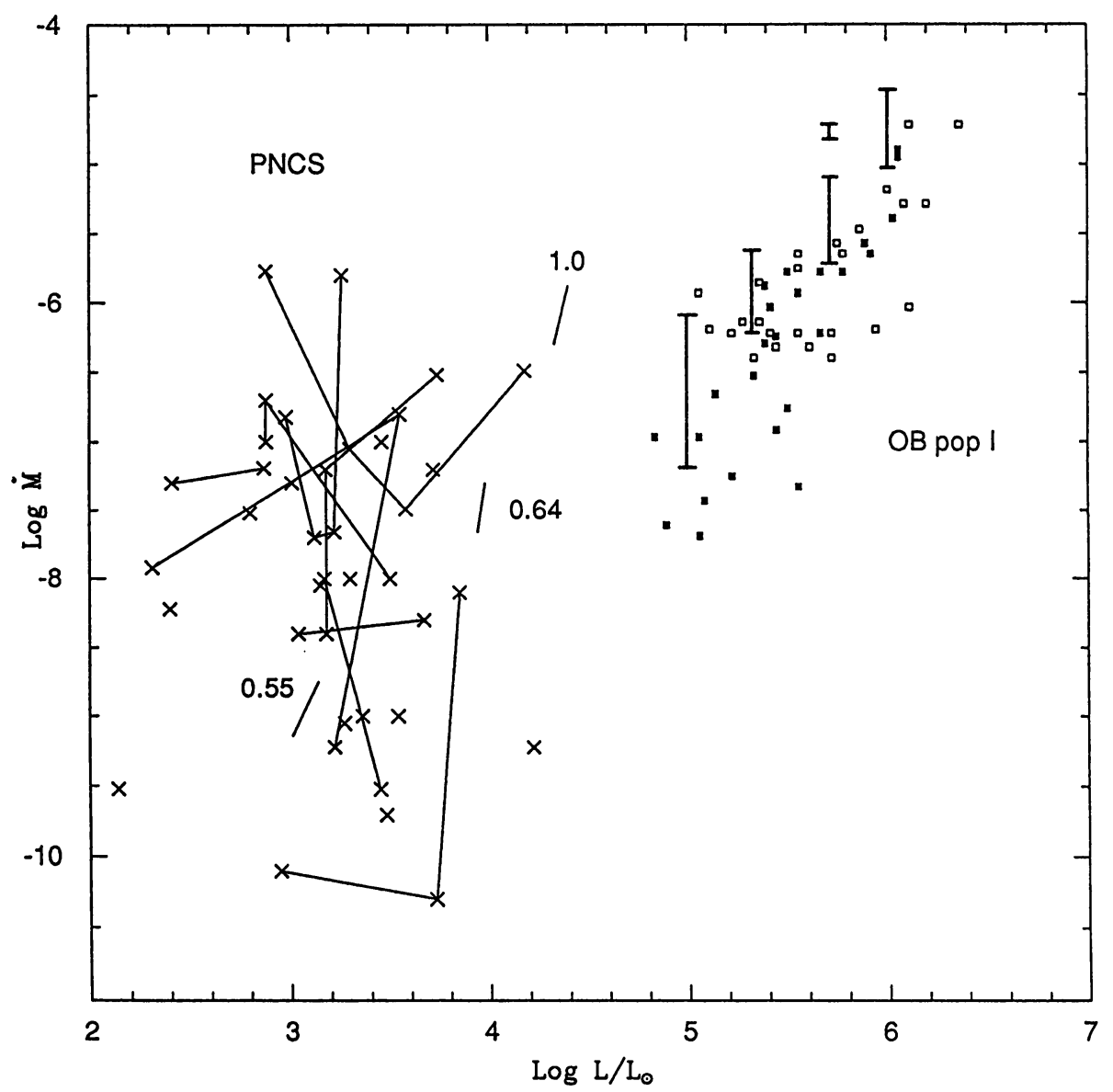

Fig 1. $\log (\dot{M})\left[M_{O} /\right.$ yr $]$ vs. $\log \left(L / L_{o}\right)$ for central stars of planetary nebulae (crosses) and for population I OB stars (squares). The last from Garmany and Conti (1984). Values belonging to the same object are joined by a straight line. Vertical bars are theoretical predictions by Friend and Abbott (1986). Tilted lines, labelled by stellar mass in $\mathrm{M}_{\mathrm{O}}$, are predictions for PNCS by Pauldrach et al. (1987). 
A satisfactory theory, lacking only of the Auger ionization, has been worked out by Kudritzki and collaborators. Then we expect that the errors in $q_{j}$ will be reduced in the future. In some of the available determinations the authors have calculated $\mathrm{q}_{\mathrm{i}}$ using only radiative processes in atoms with the ground level and the continuum only. That is clearly inaccurate, as has been dicussed by CLS and CP. Most of the authors have however used the suggestion of CLS: $q(O I V)+q(O V)=1$ or have searched for the maximum of $q \dot{M}$ across the wind. These last assumptions should not be very imprecise. By adopting the abundances measured in the nebula as representative of those in the winds (as done by most authors) one should not introduce a serious error. This if the lines of oxygen are used. The evolutionary theory in fact does not predict significant changes in the abundance of this element during the evolution of the central star. More severe is the problem of the stellar radius, where an error of factor of 2 can be introduced mostly by the uncertanity in the distance. Finally a very serious uncertanity may occur due to the assumed $\mathrm{T}_{\mathrm{rad}}$. For instance in NGC 6543 the different choices of $\mathrm{T}_{\mathrm{rad}}$ introduce a difference in the determinations of $\dot{M}$ by CLS and by PCL of a factor of 10 . To illustrate how much of the scatter of individual determinations of M may be attributed to the choice of the fundamental stellar parameters and how much may be intrinsic to the method, I consider the case of one of the best studied object: NGC 6543. In this object there are 5 values of $M$ equal to $910^{-8}, 710^{-7}, 3.210^{-7}, 1.710^{-6}, 3.210^{-8} \mathrm{M}_{\mathrm{o}} \mathrm{yr}^{-1}$ by the authors CLS, He, BCG, HS and PCL respectively. The authors have used the FM, EP, EP, FMm, and the SEI method, respectively. The first and the fourth work are made with low resolution, the others with high resolution IUE data. If, by exercise ${ }_{3}$ we use in all these studies the same fundamental stellar parameter adopted by PCL, the M of CLS and HS becomes $1.210^{-8}$ and $9.110^{-8}$, respectively. It was not simple to reduce $M$ from BCG to the parameters of PCL because of the particular treatment of the ionization by BCG and because of other complications. The same for the value by He because of lack of data in her study.

The difference between $9.110^{-8}$ obtained by HS with low resolution (after reduction to the parameters of PCL) and $3.210^{-8}$ by PCL, with high resolution IUE data and the SEI method, illustrates the residual scatter, due to the different methods, in this object. Part of this difference is due to the use of "average" velocity and opacity laws by HS with respect to the more precise ones used by PCL. Part may be due to the fact that the theory of line formation used by HS is entirely radiative while PCL have considered also collisional effects, which in this object have resulted not negligible.

How accurate is the $3.210^{-8}$ value of PCL remains an open question, largely linked to the choice of the fundamental stellar parameters. I come to this point in the next section.

\section{PREDICTION OF MULTI-SCATTERING LINE RADIATION WIND DRIVEN THEORY}

It has been noticed by CLS that the observed $\dot{\mathrm{M}}$ in NGC 6543 exceeds the limit $\dot{\mathrm{M}}_{\mathrm{max}}=\mathrm{L} /\left(\mathrm{v}_{\infty}, \mathrm{c}\right)$ implied by the single scattering line radiation wind driven theory. Lucy and Perinotto (1987; LP) have investigated whether the problem might be alleviated by the use of multi-scattering theory.

The theoretical treatment uses a Monte Carlo technique similar to that sucessfully used by Abbott and Lucy (1985) to study the wind of $\zeta$ Puppis. The analysis is simplified e.g. in the ionization structure across the wind, considered to occur only from the ground levels. However there are compenstion effects, because the wind is driven by 
some 20000 lines of several ions. Therefore, an overestimate of the abundance of a particular ion resulting in an increasing efficiency of the driving due to its lines is compensated by the contemporary underestimate of the abundance of other ions of the same element.

Input parameters are the stellar mass, the luminosity, $\mathrm{T}_{\text {eff }}, \mathrm{T}_{\mathrm{rad}}$, the elemental abundances, the velocity law of the wind and $\mathrm{v}_{\infty} . \mathrm{T}_{\mathrm{rad}}$ refers to the far UV part of the spectrum. Outputs are $\dot{M}$ and the synthesized spectrum of the wind. $\dot{M}$ is little sensitive to the velocity law.

When the fundamental stellar parameters of CLS are used, the theoretical predicted $\mathrm{M}$ is $\approx 10^{-9} \mathrm{M}_{\mathrm{O}} \mathrm{yr}^{-1}$ which is much smaller than observed by CLS (and by all the other authors). Moreover the computed spectrum does not match the observed one. By variating the parameters of the central star of NGC 6543, LP finds in the $\log \left(\mathrm{L} / \mathrm{L}_{\mathrm{O}}\right)$, $\log \mathrm{T}_{\text {eff }}$ plane a trajectory, which is approximately a straight line. Each point of it represents a solution which is both dynamically and spectroscopically consistent, i.e. the predicted spectrum resembles the observed one. The reproduction of the observed spectrum is better for points close to $\log \mathrm{T}_{\text {eff }}=4.7, \log \left(\mathrm{L} / \mathrm{L}_{\mathrm{Q}}\right)=4.0$. Along this trajectory the corresponding $\dot{M}$ increases from $2.210^{-9}$ at $\log \left(\mathrm{L} / \mathrm{L}_{\mathrm{O}}\right)=3.1$ to $10^{-7}$ when $\log \left(\mathrm{L} / \mathrm{L}_{\mathrm{O}}\right)=4.7$.

A further constraint can be obtained along the trajectory. At each point one has $\mathrm{L}$ and $\mathrm{T}_{\text {eff }}$. Thus the bolometric correction can be evaluated and then the distance of the object. From the measured expansion velocity one deduces an expansion age which can be compared with the evolutionary age in the Schonberner tracks.

A final solution is thus obtained for the central star of NGC 6543 : $\log \left(\mathrm{L} / \mathrm{L}_{\mathrm{o}}\right)=3.8, \log \mathrm{T}_{\text {eff }}=4.7, \mathrm{M}=0.62 \mathrm{M}_{\mathrm{O}}, \dot{\mathrm{M}}=1.210^{-8}$.

The solution found, totally independent, by PCL is much closer to the above than those of CLS, HS and BCG. I recall that the observational $\dot{M}$ of PCL is $3.210^{-8}$ $\mathrm{M}_{\mathrm{o}} \mathrm{yr}^{-1}$.

\section{CONCLUSIONS}

There are determinations of mass loss rates from fast winds in about 20 central stars of planetary nebulae, ranging from $510^{-11}$ to $210^{-6} \mathrm{M}_{\mathrm{o}} \mathrm{yr}^{-1}$. The scatter among values of different authors in the same object may amount to factors $10-100$.

Evidence is given that the multi-scattering line radiation wind driven theory is able to predict the observed $\dot{M}$ in a well studied object to within a factor of three. This fact may also suggest that with a careful use of the best existing methods, the observational determination of $\dot{M}$ may reach a similar accuracy .

Work needs to be done to provide values of $\dot{M}$ which may be believed to be accurate to this level in all the central stars of planetary nebulae with good quality high resolution IUE spectra, both for a close comparison with the theoretical predictions and for clarifying the role of fast winds in the life of individual nebulae and of their central stars.

\section{REFERENCES}

Abbott, D.C. and Lucy, L.B. 1985, Ap.J. $\underline{288}, 679$ 
Adam, J. and Koppen, S. 1985, Astron. Astrophys. 142, 461 (AK)

Adams, S., Seaton, M.J., Howarth, I.D., Aurière, M. and Walsh, J.R. 1984, M.N.R.A.S. 207, 471 (ASHAW)

Bianchi, L., Cerrato, S. and Grewing, M. 1986, Astron. Astrophys. 169, 227 (BCG)

Bombeck, G., Koppen, J. and Bastian, U. 1986, in ESA SP-263, p. 287 (BKB)

Castor, J.I. 1970, M.N.R.A.S. $\underline{149}, 111$

Castor, J.I. and Lamers, H.J.G.L.M., 1979, Ap. J. Suppl. 39, 481

Castor, J.I., Lutz, J.H. and Seaton, M.J. 1981, M.N.R.A.S. 194, 547 (CLS)

Cerrato, S., Baessgen, M., Bianchi, L. and Grewing, M. 1987, preprint (CBBG)

Cerruti-Sola, M. and Perinotto, M. 1985, Ap.J. 291, 237 (CP)

De Freitas Pacheco, J.A. and Veliz, J.G. 1987, M.N.R.A.S. 227, 773 (FV)

Friend, D.B., and Abbott, D.C. 1986, Ap.J. 311, 701

Garmany, C.D., Conti, P.S. 1984, Ap.J. 284, 705

Hamann, W.-R. 1980, Astron. Astrophys. 84,342

1981, Astron. Astrophys. 93, 353

Hamann, W.-R., Kudritzki, R.-P., Mendéz, R.H. and Pottasch, S.R. 1984, Astron. Astrophys. 139, 459 (HKMP)

Harrington, J.P. 1982, in 'Advances in UV Astronomy: Four Years of IUE Research' (Nasa CP-2238), p.610(H)

Heap, S. 1981 in 'The Universe at Ultraviolet Wavelengths' (NASA CP-2171), p.415 (He)

Hutsemékers, D. and Surdej, J. 1987, Astr. Astrophys. 173, 101 (HS)

Klein, R.I., and Castor, J.I. 1978, Ap.J.220, 902

Lamers, H.J.G.L.M., Cerruti-Sola, M. and Perinotto, M. 1987, Ap.J. $\underline{314}, 726$

Lucy, L.B. 1971, Ap.J. 163, 95

Lucy, L.B. and Perinotto, M. 1987, Astron. Astrophys., in press (LP)

Mihalas, D., Kunasz P.B. and Hummer D.G. 1975, Ap.J. 202, 465

Olson, G.L. 1981, Ap.J. 248, 1021 1982, Ap.J. 255,267

Pauldrach, A., Kudritzki, R.P., Gabler, R. and Wagner, A. 1987, preprint

Perinotto, M., Benvenuti, P. and Cerruti-Sola, M. 1982, Astron. Astrophys. 108 , 314 (PBC)

Perinotto, M., Cerruti-Sola M. and Lamers, H.J.G.L.M. 1987, in preparation (PCL)

Pottasch, S.R., Gathier, R., Gilra, D.P., and Wesselins, P.R. 1981, Astron. Astrophys. 102,237 (PGGW)

Schonberg, K. 1985 a, Astron. Astrophys. Suppl. 62, 339

1985 b, Astron. Astrophys. 148,405

Surdej, J. 1982, Astrophys. Space Sci. $\underline{88}, 31$

1983, Astron. Astrophys. 127, 304

1985, Astron. Astrophys. 152,361

1987, private communication

Wessolowski, U. and Hamann, W.-R. 1986, Astron. Astrophys. 167, 106 